\title{
Regular self-motion of a liquid droplet powered by the chemical marangoni effect
}

$\operatorname{AUTHOR}(\mathrm{S}):$

Nagai, Ken; Sumino, Yutaka; Yoshikawa, Kenichi

\section{CITATION:}

Nagai, Ken ... [et al]. Regular self-motion of a liquid droplet powered by the chemical marangoni effect. Colloids and Surfaces B: Biointerfaces 2007, 56(1-2): 197-200

\section{ISSUE DATE:}

2007-04

URL:

http://hdl.handle.net/2433/49164

\section{RIGHT:}

Copyright (C) 2007 Elsevier B.V. All rights reserved.; This is not the published version. Please cite only the published version.; この論文は 出版社版でありません。引用の際には出版社版をご確認ご利用くださ い。 


\title{
Regular Self-Motion of a Liquid Droplet Powered by the Chemical Marangoni Effect
}

\author{
Ken Nagai, Yutaka Sumino and Kenichi Yoshikawa \\ Department of Physics, Graduate School of Science, Kyoto University, \\ Kyoto 606-8502, Japan \\ E-mail: yoshikaw@scphys.kyoto-u.ac.jp \\ Received XXXXth Month, 200X \\ Accepted XXXXth Month,200X \\ DOI: $10.1039 /$
}

We describe here our recent work on spontaneous regular motion of liquid droplet powered by the chemical Marangoni effect under spatially symmetric conditions. It is shown that a spontaneously crawling oil droplet on a glass substrate with a nonequilibrium chemical condition of cationic surfactant exhibits regular rhythmic motion in a quasi-one-dimensional vessel, whereas irregular motion is induced in a two-dimensionally isotropic environment. Such behavior of a droplet demonstrates that spontaneous regular motion can be generated under fluctuating conditions by imposing an appropriate geometry. In a simpler liquid/liquid system under isotropic environment, an alcohol droplet exhibits spontaneous motion on water layer. The droplet spontaneously forms a specific morphology depending on its volume, causing specific mode of translational motion. An alcohol droplet with a smaller volume floating on water surface moves irregularly. On the other hand, a droplet with a larger volume undergoes vectorial motion accompanied by deformation into an asymmetric shape. This result suggests a scenario on the emergence of regular motion coupled with geometrical pattern formation under far-from-equilibrium conditions.

Keywords: Marangoni effect; Interfacial tension; Spontaneous motion; Droplet; Nonequilibrium

\section{Introduction}

Under equilibrium conditions, any macroscopic spatio-temporal structure can not be generated. On the other hand, in nonequilibrium open system, uniform stationary state can be unstable and spatio-temporal pattern can spontaneously emerge, i.e., dissipative structure [1]. As a kind of dissipative structures, it is known that macroscopic agitation of interface occurs when two different liquid systems contact each other [2-6]. Such self-agitation is called the chemical Marangoni effect [7]. As a phenomenon related to the chemical Marangoni effect, spontaneous motion of alcohol-water mixture, tears of wine, has been found more than 150 years ago $[8,9]$.

The chemical Marangoni effect can induce the motion of an object with mm-cm scale. In such scale, the effect of the interface becomes dominant compared with other volume effect such as inertia; therefore, the chemical Marangoni effect can trigger the spontaneous translational motion of an object [10]. It is reported that making system spatially asymmetric enables the object to exhibit regular motion by the Marangoni effect [11-13]. Whereas, in spatially symmetric environment, the spontaneous motion powered by the chemical Marangoni effect appears to be irregular in general [14-20]. As the exception, occurrence of rhythmic or directional motion has been encountered in some experiments with 
spatially symmetric vessel [21-23], whose mechanism has not been confirmed yet. Recently, we have found that spontaneous translational motion of a droplet changes from irregular to regular depending on geometrical constraints or the volume of a droplet. It has become clear that the shape of the boundary is essential to extract the regular motion induced by the chemical Marangoni effect. It has also been found that spontaneous deformation of a droplet can induce the appropriate boundary condition for the regular translational motion of a droplet.

\section{Emergence of regular motion in a confined geometry}

Figure 1 exemplifies the spontaneous motion of an oil droplet on a glass substrate in an aqueous phase. Immediately after it was transferred to the glass substrate, an oil droplet began to move driven by a difference in interfacial tension. As shown in Fig. 1(a), an oil droplet exhibited random agitation in a petri dish. In contrast to this, regular rhythmic motion was generated in the same chemical system simply by changing the spatial geometry, as shown in Fig. 1(b). Corresponding movies are available online [24].

[Figure 1 around here.]

The generation of a difference in interfacial tension between the front and the rear side can be explained as schematically shown in Fig. 2. Since $\mathrm{STA}^{+}$(stearyl trimethyl ammonium ion) is aggregated on the glass surface, the glass surface becomes hydrophobic. $\mathrm{STA}^{+}$attached to the glass surface tends to dissolve into the oil droplet; hence, the glass surface under an oil droplet becomes more hydrophilic. Once an oil droplet starts to move, the glass surface becomes less hydrophobic at the rear side of the droplet and the difference in interfacial tension is generated between the front and rear side of the droplet. This imbalance in the interfacial tension promotes directed motion against viscous damping. Significantly, the surface of the glass substrate spontaneously returns to the hydrophobic state soon after an oil droplet passes by since $\mathrm{STA}^{+}$in the aqueous phase tends to be absorbed onto the glass substrate. This mechanism allows the trajectory of an oil droplet to cross itself; thus, rhythmic and repetitive motion can be generated in this system. This is in contrast with the past theoretical model of droplet moving spontaneously, whose trajectry cannot cross $[14,25]$.

[Figure 2 around here.]

Let us now discuss the mechanism of the occurrence of regular, rhythmic motion in this selfagitating system. The characteristic features of the motion of an oil droplet can simply be described as,

$$
\frac{d \boldsymbol{v}}{d t}=\eta v_{0}^{2} \boldsymbol{v}-\eta|\boldsymbol{v}|^{2} \boldsymbol{v}+\boldsymbol{\xi}(t),
$$

where $\eta$ and $v_{0}$ are constant [26]. The first term represents the driving force caused by the difference in the interfacial tension, where we incorporate the characteristics of self-motion prohibiting backward motion as in Fig. 1. The second term represents resistance. The third term $\boldsymbol{\xi}$ represents the white random force simplifying the effect of the inhomogeneity in the glass surface. The fixed points of Eq. 1 are $|\boldsymbol{v}|=v_{0}, 0$, where $|\boldsymbol{v}|=0$ is an unstable fixed point. When the system is two-dimensional, the attractor in velocity space, i.e., the circle $|\boldsymbol{v}|=v_{0}$, is neutrally stable with regard to their angular orientation. On the other hand, there are two stable fixed points $\left(\boldsymbol{v}=v_{0},-v_{0}\right)$ in a one-dimensional system. Therefore, the droplet spontaneously moves regularly in a quasi-one-dimensional system in contrast to the irregular motion in a twodimensional system [27].

\section{Mode switching depend- ing on the size of droplet}

[Figure 3 around here.]

[Figure 4 around here.] 
Figure 3 shows the spontaneous motion of an alcohol droplet on an aqueous phase. When the volume of the droplet was less than $0.1 \mu \mathrm{l}$, the droplet shape is circular and the droplet moved irregularly as shown in Fig. 3(b). This motion resembled that of a crawling oil droplet in a two-dimensional system shown in Fig. 1(a). On the other hand, a droplet with a volume of $10 \mu \mathrm{l}$ spontaneously assumed an asymmetric shape and exhibited vectorial motion as shown in Fig. 3(c). Figure 3(d) shows a phase diagram of the mode of motion with a change in volume. Without any changes in the external boundary condition, a droplet powered by the chemical Marangoni effect exhibited regular motion when the size of the droplet exceeded a certain threshold. Corresponding movies are available online [24].

The spontaneous motion of the smaller droplet can be understood as follows. Once the droplet with circular shape begins to move, the alcohol concentration gradient becomes larger in the front side than in the rear side. As interfacial tension monotonically decreases with the increase of the alcohol concentration, the interfacial tension gradient becomes larger in the front side than in the rear side. This situation causes asymmetric convective flow, which allows the droplet to continue its motion, as shown in Fig. 4(a). It is to be noted that the motion is irregular, similar to the crawling oil droplet in a two-dimensional system [20]. In contrast, the larger droplet moves vectorially. The experimental trend in volume-dependent modeswitching is explained in terms of the competition between the characteristic wave-length and the length scale of the droplet as follows (Fig. 4(b), (c)).

Alcohol molecules diffuse across the triple line toward the water surface. Around the triple line with a higher curvature, the concentration gradient is higher, which results in a higher interfacial tension gradient. This difference in the interfacial tension gradient causes growth of the small deformation at the triple line. There exists a characteristic wave-length of deformation where the force that promotes the deformation is equal to the interfacial tension that dampens the deformation. When the wave-length of the deformation is smaller than the characteristic wave-length, the deformation is suppressed. As the length of the triple line is slightly larger than the characteristic length, only the fundamental wave, i.e., lowest wave number, around the droplet can grow; as a result, the droplet exhibits morphological deformation as in Fig. $3(\mathrm{c})$. Once the droplet is deformed, the variation in curvature of the triple line causes spatial asymmetry in the interfacial tension gradient. This asymmetric distribution of pentanol induces the asymmetric convective flow which drives the directional motion of the droplet from the concave region to the convex region, as illustrated in Fig. 4(c) [28].

\section{Conclusion}

We have experimentally found that the spontaneous motion of a droplet powered by the chemical Marangoni effect becomes regular in spatially symmetric systems under suitable geometrical shape of vessel. In addition, we have also shown that spontaneous deformation of liquid boundary by the chemical Marangoni effect can trigger regular motion without an external change in boundary.

It is expected that our works inspire the study to abstract the essence of the mechanism of selfmotion in biological system.

\section{Acknowledgement}

We thank Prof. M. Nagayama (Kanazawa Univ.), Prof. S. Nakata (Nara Univ. of Edu.), Dr. N. Magome (Nagoya Bunri College) and Dr. H. Kitahata (Kyoto Univ.) for their helpful discussions. This research was partially supported by Grants-in-Aid for Scientific Research for Kenichi Yoshikawa (18651072 and 18GS0421) and for JSPS Fellowships for Ken Nagai (18-3189) and for Yutaka Sumino (183188) from the Ministry of Education, Culture, Sports, Science and Technology of Japan.

Ken Nagai, Yutaka Sumino and Kenichi 


\section{Yoshikawa}

Department of Physics, Graduate School of Science, Kyoto University, Kyoto 606-8502, Japan.

E-mail: yoshikaw@scphys.kyoto-u.ac.jp 


\section{References}

[1] G. Nicolis and I. Prigogine, Selforganization in nonequilibrium systems : from dissipative structures to order through fluctuations, Wiley, New York, 1977.

[2] M. Dupeyrat and E. Nakache, Bioelectrochem. Bioenerg., 1978, 5, 134.

[3] E. Nakache, M. Dupeyrat, and M. VignesAdler, J. Colloid, 1983, 94, 187.

[4] M. Santiago-Rosanne, M. Vignes-Adler, and M. G. Velarde, J. Colloid Interface Sci., 1997, 191, 65.

[5] V. I. Kovalchuk, H. Kamusewitz, D. Vollhardt, and N. M. Kovalchuk, Phys. Rev. E, 1999, 60, 2029.

[6] ed. T. S. Sørensen; Dynamics and Instability of Fluid Interfaces, 1978.

[7] L. E. Scriven and C. V. Sternling, Nature, 1960, 187, 186.

[8] J. B. Fourbier and A. M. Cazabat, Europhys. Lett., 1992, 20, 517.

[9] J. Thomson, Phil. Mag., 1855, 10, 330.

[10] C. D. Bain, G. D. Burnett-Hall, and R. R. Montgomerie, Nature, 1994, 372, 414.

[11] M. K. Chaudhury and G. M. Whitesides, Science, 1992, 256, 1539.

[12] K. Yoshikawa and N. Magome, Bull. Chem. Soc. Jpn., 1993, 66, 3352.

[13] S. Nakata, Y. Iguchi, S. Ose, M. Kuboyama, T. Ishii, and K. Yoshikawa, Langmuir, 1997, 13, 4454.

[14] F. D. D. Santos and T. Ondarçuhu, Phys. Rev. Lett., 1995, 75, 2972.

[15] S. Nakata and Y. Hayashima, J. Chem. Soc., Faraday Trans., 1998, 94, 3655.

[16] A. K. Schmid, N. C. Bartelt, and R. Q. Hwang, Science, 2000, 290, 1561.
[17] N. Watanabe, K. Kutsumi, and O. Sano, J. Phys. Soc. Jpn., 1994, 63, 2955.

[18] H. Gruler, Liquid Crystals, 1998, 24, 49.

[19] N. Magome and K. Yoshikawa, J. Phys. Chem., 1996, 100, 19102.

[20] A. S. Mikhailov and V. Calenbuhr, From Cells to Societies, Springer, Berlin, 2002.

[21] S. Bekki, M. Vignes-Adler, E. Nakache, and P. M. Adler, J. Colloid Interface Sci., 1990, 140, 492.

[22] S. Bekki, M. Vignes-Adler, and E. Nakache, J. Colloid Interface Sci., 1992, 152, 314 .

[23] O. Sano, K. Kutsumi, and N. Watanabe, J. Phys. Soc. Jpn., 1995, 64, 1993.

[24] http://www.chem.scphys.kyotou.ac.jp/movies/self-movingdroplet/index_e.html.

[25] F. Brochard, Langmuir, 1989, 5, 432.

[26] U. Erdmann, W. Ebeling, L. SchimanskyGeier, and F. Schwitzer, Eur. Phys. J. B, 2000, 15, 105.

[27] Y. Sumino, N. Magome, T. Hamada, and K. Yoshikawa, Phys. Rev. Lett., 2005, 94, 068301.

[28] K. Nagai, Y. Sumino, H. Kitahata, and K. Yoshikawa, Phys. Rev. E, 2005, 71, 065301. 


\section{Figure Captions}

Fig. 1

(a) Spontaneous motion of an oil droplet in a two-dimensional system. Left: Experimental setup. Right: Irregular motion appeared under spatially symmetric conditions. (b) Spontaneous motion in a quasi-one-dimensional system. Left: Experimental setup. Right: Periodic forwardand-backward motion was generated on a narrow, straight glass substrate. In both experiments, the aqueous phase was water with stearyl trimethyl ammonium chloride (STAC), and the organic phase was an iodine solution of nitrobenzene saturated with potassium iodide.

Fig. 2

Mechanism of spontaneous motion of an oil droplet. Red arrows show the direction of oildroplet motion. $\mathrm{STA}^{+}$dissolves into the oil droplet from the glass surface. Thus, the difference in interfacial tension is generated, inducing the motion of the oil droplet. The glass surface returns to hydrophobic shortly after the oil droplet passes by since $\mathrm{STA}^{+}$in the aqueous phase again adheres to the glass surface.

\section{Fig. 3}

(a)Experimental system. (b)Irregular motion of a smaller droplet $(0.02 \mu \mathrm{l})$. (c)Left: Vectorial motion of a larger droplet $(10 \mu \mathrm{l})$. Right: Morphology of the droplet at $3.0 \mathrm{~s}$. (d)Phase diagram of the mode of spontaneous motion. $\square$, irregular motion and $\boldsymbol{\bullet}$, vectorial motion. When the volume exceeds $300 \mu \mathrm{l}(\mathrm{O})$, the droplet breaks into smaller droplets. The aqueous phase contained $2.3 \mathrm{vol} \%$ pentanol and alcohol phase is pentanol stained with ink to visualize the droplet.

Fig. 4

Mechanism of the mode switching in Fig. 3. (a)Spontaneous motion of an alcohol droplet with circular shape. White arrows represent strength of the Marangoni flow. Upper: When the droplet does not move, the concentration distribution at an air-water interface is symmetric. Lower: When the droplet moves toward a certain direction, the concentration gradient of alcohol in the front side becomes larger than that in the rear side; thus, asymmetric convective flow is induced, which makes the droplet continue to move. The green arrow represents the direction of the droplet motion. (b)Real part of eigenvalue of the perturbation with the wave-number $k$ along the triple line among alcohol, water and air phases. There exists a critical wave-number $k_{\mathrm{c}}$, below which the amplitude grows with time. Using a linear stability analysis, the instability is characterized by the eigenvalue of each normal mode. The smallest wave-number, $k_{0}$, corresponding to the largest wave-length, is dependent on the length of the triple line, $L$ or the volume of the droplet, $V$ : $k_{0} \sim L^{-1} \sim V^{-\frac{1}{3}}$. (c)Concentration distribution of alcohol at water-air interface around a droplet with the shape in Fig. 3(c). The horizontal line represents the coordinate along the solid line in the bottom figure. The difference of the concentration gradient between front and rear sides causes the asymmetric convective flow, which drives the vectorial motion of the alcohol droplet. 
(a)

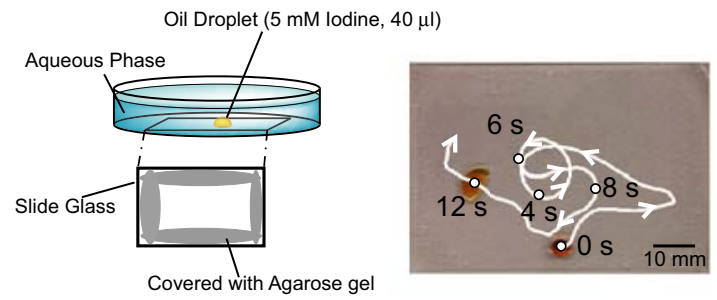

(b)

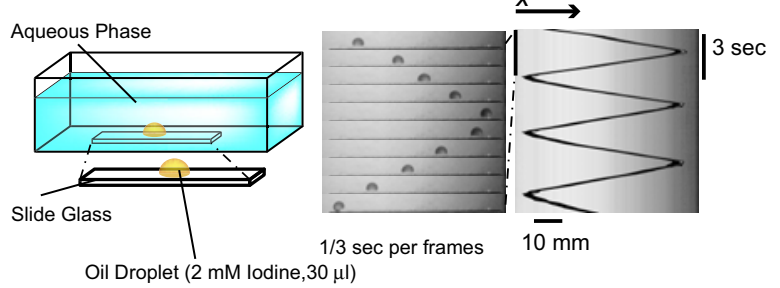

Figure 1: (a) Spontaneous motion of an oil droplet in a two-dimensional system. Left: Experimental setup. Right: Irregular motion appeared under spatially symmetric conditions. (b) Spontaneous motion in a quasi-one-dimensional system. Left: Experimental setup. Right: Periodic forward-and-backward motion was generated on a narrow, straight glass substrate. In both experiments, the aqueous phase was water with stearyl trimethyl ammonium chloride (STAC), and the organic phase was an iodine solution of nitrobenzene saturated with potassium iodide. Color version of this figure is in pdf version of this article. 

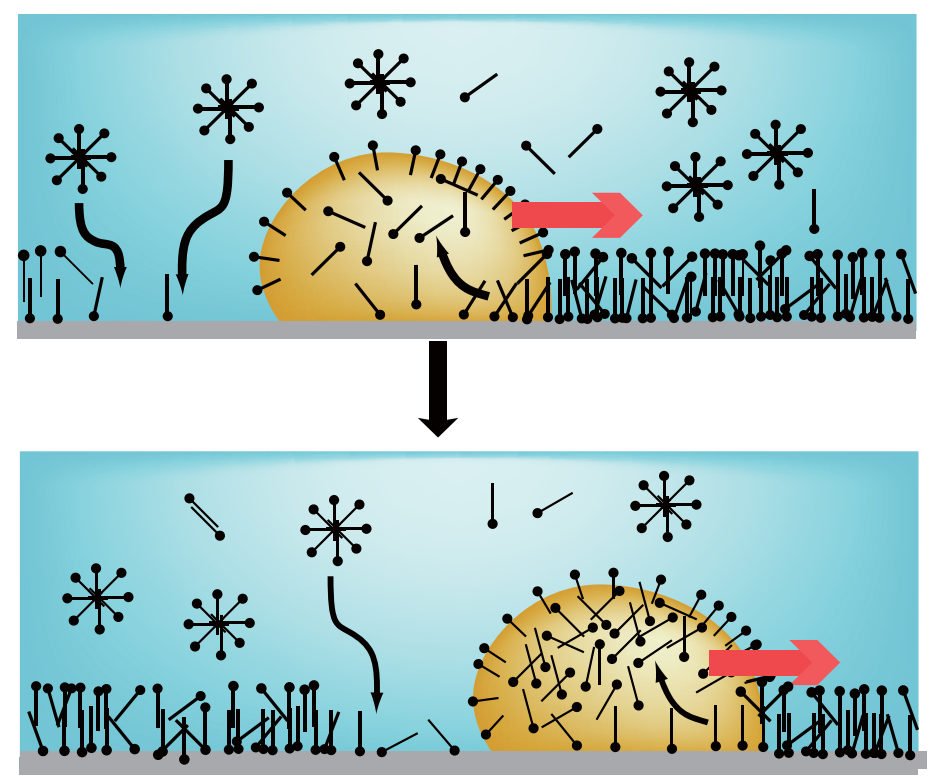

Figure 2: Mechanism of spontaneous motion of an oil droplet. Red arrows show the direction of oil-droplet motion. $\mathrm{STA}^{+}$dissolves into the oil droplet from the glass surface. Thus, the difference in interfacial tension is generated, inducing the motion of the oil droplet. The glass surface returns to hydrophobic shortly after the oil droplet passes by since $\mathrm{STA}^{+}$in the aqueous phase again adheres to the glass surface. Color version of this figure is in pdf version of this article. 
(a)

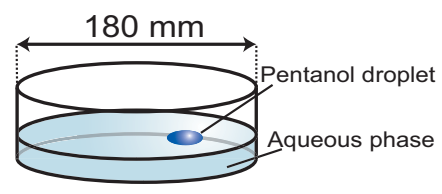

(c)

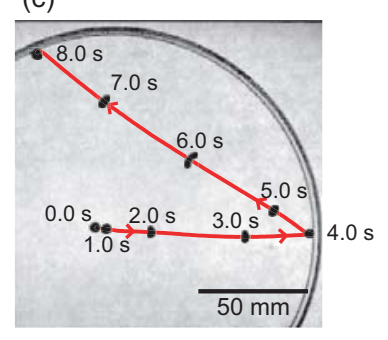

(d)

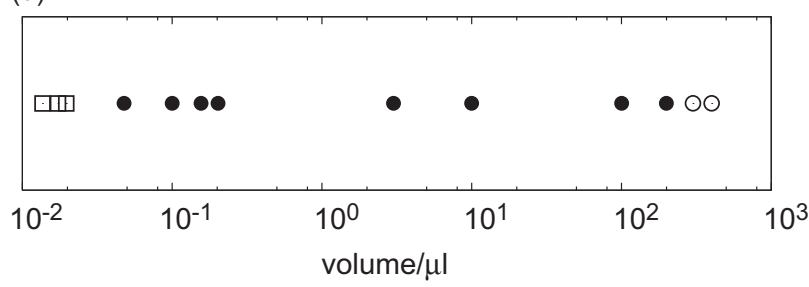

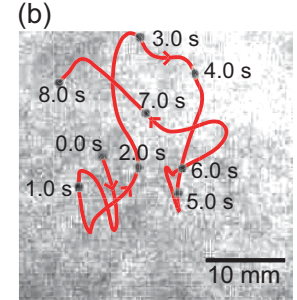

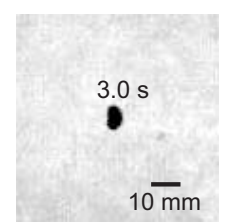

$10 \overline{\mathrm{m} m}$

Figure 3: (a)Experimental system. (b)Irregular motion of a smaller droplet $(0.02 \mu \mathrm{l})$. (c)Left: Vectorial motion of a larger droplet $(10 \mu \mathrm{l})$. Right: Morphology of the droplet at $3.0 \mathrm{~s}$. (d)Phase diagram of the mode of spontaneous motion. $\square$, irregular motion and $\boldsymbol{\bullet}$, vectorial motion. When the volume exceeds $300 \mu \mathrm{l}(\mathrm{O})$, the droplet breaks into smaller droplets. The aqueous phase contained $2.3 \mathrm{vol} \%$ pentanol and alcohol phase is pentanol stained with ink to visualize the droplet. Color version of this figure is in pdf version of this article. 


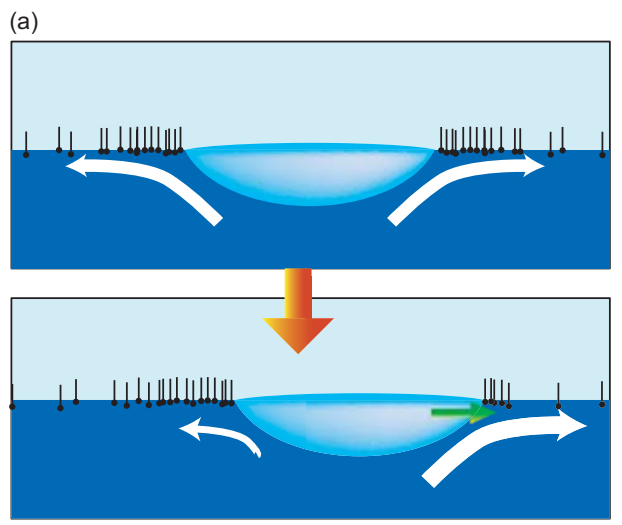

(b)

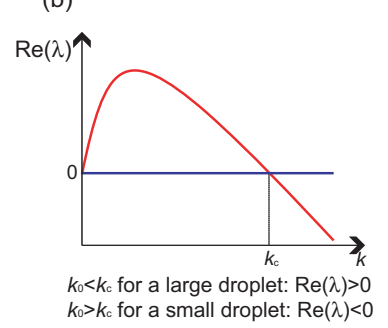

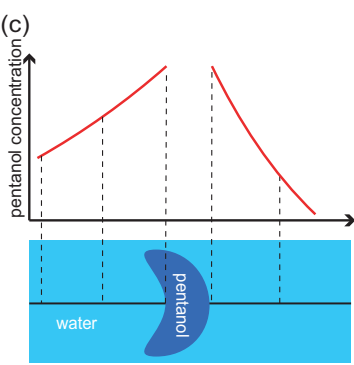

Figure 4: Mechanism of the mode switching in Fig. 3. (a)Spontaneous motion of an alcohol droplet with circular shape. White arrows represent strength of the Marangoni flow. Upper: When the droplet does not move, the concentration distribution at an air-water interface is symmetric. Lower: When the droplet moves toward a certain direction, the concentration gradient of alcohol in the front side becomes larger than that in the rear side; thus, asymmetric convective flow is induced, which makes the droplet continue to move. The green arrow represents the direction of the droplet motion. (b)Real part of eigenvalue of the perturbation with the wavenumber $k$ along the triple line among alcohol, water and air phases. There exists a critical wave-number $k_{\mathrm{c}}$, below which the amplitude grows with time. Using a linear stability analysis, the instability is characterized by the eigenvalue of each normal mode. The smallest wave-number, $k_{0}$, corresponding to the largest wave-length, is dependent on the length of the triple line, $L$ or the volume of the droplet, $V: k_{0} \sim L^{-1} \sim V^{-\frac{1}{3}}$. (c)Concentration distribution of alcohol at waterair interface around a droplet with the shape in Fig. 3(c). The horizontal line represents the coordinate along the solid line in the bottom figure. The difference of the concentration gradient between front and rear sides causes the asymmetric convective flow, which drives the vectorial motion of the alcohol droplet. Color version of this figure is in pdf version of this article. 